\title{
La globalización de las ciudades. Integración Regional desde la gestión local
}

\author{
Bilbao Terk, Miriam*
}

\section{Resumen}

La globalización ha producido la modificación del espacio local a partir de una nueva articulación de la relación espacio tiempo. Por tanto ha generado espacios y relaciones diferentes a las conocidas, afectando más directamente a aquellas ciudades que por su ubicación geográfica relativa, ta maño y complejidad de sus funciones reciben un impacto más directo del fenómeno. Esta relocalización acarrea diversas consecuencias, pero en particular la instalación de un nuevo escenario con actores visibles y actores virtuales que cruzan con sus decisiones el espacio de la ciudad. Los problemas locales se convierten en problemas globales Como contrapartida, los gobiernos locales deben afrontar esta agenda globalizada con los mismos instrumentos y recursos que disponían cuando su acción se limitaba a los servicios públicos esenciales. En consecuencia aparecen modalidades de integración que suponen nuevos tipos de gestión e interacción construyendo redes sin fronteras, traspasando y rebasando los límites y las potestades institucionales establecidas. A partir de esta perspectiva se formulan las instancias de integración y las nuevas formas de gestión en la que se encuentra inserto el espacio ciudad del municipio de Mendoza en Argentina.

Palabras clave: Globalización, integración regional, gestión local.

Recibido: 00-10-31. Aceptado: 01-10-02

Magister en Gestión de las Organizaciones Públicas. Profesora de la Facultad de Ciencias Políticas y Sociales - Universidad Nacional de Cuyo-.y Directora del Proyecto de Investigación Nuevas Tecnologías para la Gestión del Empleo en la Facultad de Ciencias Económicas y Facultad de Ciencias Políticas y Sociales de la Universidad Nacional de Cuyo (Argentina). E-Mail: bilbaoterk@arnet.com.ar.

Colaborador: Olmedo, Rodrigo Luis. Estudiante de Ciencias Políticas y Administración Pública de la Facultad de Ciencias Políticas y Sociales. Universidad Nacional de Cuyo. Integrante del equipo de investigación Proyecto "Nuevas Tecnologías para la Gestión del Empleo. 


\section{The Globalization of Cities. Regional Integration Through Local Action}

\section{Abstract}

Globalization has produced modifications of local space through a new articulation of the space time relationship. For this reason, it has generated different spaces and relations from those previously known, directly affecting those cities which due to their relative geographic locations, size, and the complexity of their functions, receive a more direct impact due to this phenomenon. From this re-localization derive diverse consequences, but the principal one is the establishment of a new scenario with visible and virtual actors whose decisions intervene and intersect city space. Local problems become global problems. On the other hand, local governments must confront this global agenda with the same instruments and resources with which they managed essential public services. As a consequence, new models of integration have appeared which imply new types of management and interaction in the construction of networks without frontiers, going beyond and overcoming previously established institutional limits and jurisdictions. Based on this perspective, possibilities of integration and new forms of management are formulated for city space in the case of Mendoza Municipality in Argentina.

Key words: Globalization, regional integration, local management.

\section{Introducción}

Entre sus múltiples manifestaciones, la globalización ha producido la modificación del espacio local a partir de una nueva articulación de la relación espacio-tiempo. Por tanto ha generado espacios y relaciones diferentes a las conocidas, de modo que la globalización afecta más directamente algunas ciudades que por su ubicación geográfica relativa o su ubicación virtual, tamaño y complejidad de sus funciones.

Esta relocalización espacio temporal acarrea diversas consecuencias, pero en particular la instalación de un nuevo escenario con actores visibles y actores virtuales que cruzan con sus decisiones el espacio de la ciudad, convertida ahora en el locus de una red de interacciones con características de una gran heteroge- neidad (actores e instituciones locales nacionales e internacionales) y con agendas globalizadas. El desempleo, la seguridad, la contaminación, han dejado de ser en este contexto problemas locales para convertirse en cuestiones globales. Como contrapartida, al menos formal e institucionalmente, los gobiernos locales deben afrontar esta agenda globalizada con los mismos instrumentos y recursos que disponían cuando su acción se limitaba a los servicios públicos esenciales.

Esta inesperada asimetría entre demandas y recursos que, en una concepción clásica de la administración, llevaría a la reproducción de la administración central (criterio de la correspondencia orgánico - funcional en la división del trabajo), puede encontrar en el espacio local otro tipo de respuestas altamente creativas e innovadoras. En este sentido, 
queremos transmitir una serie de experiencias compartidas con un grupo de ciudades del Mercosur, todavía en ciernes en algunas de sus realizaciones, pero que muestran toda la potencialidad de las nuevas formas de gestión. Lo primero que aparece es la construcción de un nuevo espacio de acción del gobierno lacal, absolutamente ampliado en sus dimensiones, que abarca bien regiones geográficamente vecinas (salteándose en estos casos los límites provinciales o nacionales) o bien alinea ciudades con características y problemáticas comunes independientemente de su integración espacial, como en el caso de las Mercociudades. Este espacio que ha desbordado la jurisdicción político administrativa, conlleva una nueva concepción en la clásica relación estado-territorio que ha caracterizado al estado moderno desde su creación (paralelamente obliga a redefinir el concepto de espacio local como ámbito de acción de los gobiernos locales).

En consonancia con estos nuevos espacios locales (NEL) aparecen modalidades de integración que suponen fundamentalmente nuevos tipos de gestión e interacción que cruzan no sólo el espacio ciudadano sino que construyen redes sin fronteras, traspasando y rebasando los límites y las potestades institucionales establecidas.

Estas redes también pretenden reemplazar la protección que tradicionatmente brindaron las estructuras y las organizaciones conocidas, intentado superar el "sentimiento de intemperie" que deja su destrucción. A partir de esta perspectiva se describen las instancias de in- tegración y las nuevas formas de gestión en la que se encuentra inserto el espacio ciudad del municipio de Mendoza.

\section{Globalización ¿sólo un concepto económico?}

El proceso de globalización con sus características particulares, sus conceptos y definiciones, parece estar limitado al campo meramente económico, en donde hablar de regionalización, localización o descentralización administrativa del aparato estatal son conceptos exclusivos de esta disciplina, sin embargo la idea de integración cultural, ética y globalización o la idea de identidad, aparecen por lo bajo para sentar posiciones respecto de la globalización.

A tal efecto, el VI Seminario Internacional de Ciencias Sociales y Humanidades, desarrollado en el mes de enero del corriente año en la Universidad de Santiago de Chile, arrojó luz sobre los problemas de la globalización y la necesaria integración de los países latinoamericanos con respecto a áreas poco desarrolladas por los especialistas de este tema. El Dr. Christian Parker (2000), Director del Instituto de Estudios Avanzados de dicha Universidad, refiriéndose al concepto de globalización y mercado decía: “... frente a la hegemonía del mercado como mecanismo de regulación de la economía internacional, en el proceso de globalización, la integración supone una nueva agenda en las relaciones internacionales, que incorpore dimensiones adicionales a la integración comercial y financiera, es decir, que asuma aspectos vinculados con la educación, las migra- 
ciones, los grupos sociales (incluido el derecho de las mujeres y los indígenas, la lengua, la historia, la corrupción y el narcotráfico), el medio ambiente, el turismo, las artes y las letras...".

Haciendo eco de este planteo consideramos que no sólo podemos pensar en un mundo económicamente activo, con flujos de capitales, en donde encontramos un Estado desinteresado por lo social y en donde la descentralización arrastra a la crisis a los organismos más pequeños, sin recursos económicos efectivos para afrontarla, y en el cual el municipio es el encargado de dar las respuestas a los problemas planteados sino también en espacios (nuevos espacios) integrados para dar respuesta a los desafíos de la globalización.

Es necesario pensar que si queremos una verdadera participación en un estado responsable, si pretendemos considerar a los municipios como gestores de la misma y si consideramos que éstos están capacitados para sustentar el desarrollo que se plantea en este nuevo siglo, la agenda municipal debe ampliarse para incluir como prioritarios estos otros temas. Esto es posible en el marco de una ética que reconozca al individuo bajo el principio de solidaridad y respeto.

\section{La crisis de los estados nacionales: Integración Regional desde la gestión local}

En los últimos años, en América Latina, como en muchos países subdesarrollados o en "vías de serlo", se han generado una serie de transformaciones políticas, económicas y sociales que han llevado a la modificación del Estado de bienestar o desarrollista al neoliberal, privatizador o post social.

Si se analiza con más detalle este tránsito se puede observar que no se ha producido únicamente una profunda crisis en el Estado de bienestar y el intento neoliberal de su reformulación, sino que también se ha planteado la crisis del Estado-nación. Esto se evidencia en el análisis detallado que refleja la pérdida de las soberanías nacionales y la aparición de simetrías entre los diferentes países de una misma región.

El hecho es que el Estado-nación como actor soberano o autónomo por excelencia de las relaciones internacionales de los últimos tres siglos, ha entrado en crisis. El paradigma realista que sustentaba su existencia en cuanto al orden internacional ha ido variando, configurando nuevas alternativas al mismo. El paradigma de la sociedad global, se perfiló como el nuevo esquema de las relaciones internacionales, en donde nuevos actores tales como las empresas transnacionales, organismos no gubernamentales y actores sociales sin frontera emergen por sobre el estado.

\section{Descentralización/ Fragmentación}

El proceso de globalización económica y política, al que ya hemos hecho referencia, crea una red de contactos económicos que influyen cada día más en la globalización de las políticas económicas de los Estados. Pero las demandas sociales sobre los Estados y la globalización de los sistemas políticos se han incrementado, dando relevancia al tema de 
la globalización, aunque en ocasiones se puede observar que priman los objetivos, tratando de alivianar los gastos del gobierno central, por sobre la necesidad para mejorar el rendimiento del sistema político para formular e implantar políticas. Así, la globalización influye sobre el espacio y las capacidades de los gobiernos locales resaltando la fragmentación social y exigiendo nuevas formas de articulación social; determinando un modelo restrictivo de crecimiento económico, ya que sólo permite este en tanto se adapte a las demanda de los mercados globalizados. De este modo se va perfilando una retroalimentación entre los procesos internos y externos con los que debe lidiar el gobierno local.

La descentralización implica un movimiento de funciones desde los gobiernos nacionales y provinciales a los municipios y las organizaciones sociales. Se supone que este movimiento de capacidades democratizaría la sociedad, sin embargo la descentralización en el marco de la crisis fiscal del Estado de bienestar supone un mal negocio para los gobiernos locales que aumentan sus demandas en mayor proporción a sus recursos.

Esta descentralización tiene dos argumentos que la justifican; uno político social que afirma que la gestión local favorece la participación y ésta, la globalidad y la estabilidad; y otro económico, que sostiene que los gobiernos locales son más eficientes en el manejo de los recursos públicos pues están sujetos al control popular.

Entonces queda claro que las demandas y las tensiones que los estados locales deben manejar son superiores a las delegadas y plantean una serie de interrogantes que no pueden ser resueltos sin tener en cuenta a todos los actores intervinientes en el proceso (político, económico y fundamentalmente social).

Además, es necesario tener en cuenta, desde un punto de vista político, que la consolidación de la democracia se ha producido junto con una importante desafección de los electores, desprestigio institucional, inclusión precarizada o clientelar, corrupción y una especie de imposibilidad de la clase política para arbitrar modificaciones a las orientaciones principales del mercado, lo que contribuye a modificar el tipo de conflictividad que debe manejar el gobierno, a la par que ha reorientado las expectativas y demandas del ciudadano hacia el gobierno local.

El modelo único de desarrollo coloca a los municipios en la necesidad de disminuir los costos sociales y económicos y la incertidumbre, si realmente pretende insertarse en las redes globalizadas de producción.

Para aumentar las probabilidades de una inserción exitosa, la descentralización y el fortalecimiento de los NEL., deben preverse en cuanto herramientas que permiten intervenir, en mayor o menor grado, sobre algunas variables que no pueden ser modificadas por los gobiernos centrales. Un gobierno fuerte es predecible; $y$ en el contexto cambiante de la globalización, ser predecible es un recurso tan importante como la capacitación de los recursos humanos.

Pero ser predecible no es opuesto a ser innovador; para seguir siendo predecibles los NEL, deben ser innovadores insertándose en la aldea global. Estar in- 
sertados en el mundo significa producir y trabajar para el mundo y para el futuro; $y$ estar fuera de ese mundo parece significar baja calidad de servicios públicos, desempleo, emigración.

\section{Nuevos límites. Estado/ Sociedad}

El achicamiento del Estado significó que algunas de sus antiguas competencias han sido trasladadas a los ámbitos globalizados, donde los Estados nacionales y los entes locales pueden coordinar y concertar algunas prácticas con otros actores globalizadores.

Gobernar entre redes globalizadoras y sus flujos significa atenuar los efectos más graves de la inserción y de las frecuentes crisis que sufre el sistema económico internacional, también es negociar para resolver conflictos, diseñar políticas viables para el desarrollo local que estén en consonancia con las demandas del mundo globalizado.

Las modificaciones operadas por la gestión posfordista del sistema productivo han afectado también la definición de lo público y lo privado; la forma de asociatividad de las sociedad preglobalizadas cambia a partir de la exacerbación de la competencia y la disminución de las fuentes de trabajo, en las economías de escasa eficiencia. La fragmentación social trajo consigo la potenciación y dinamización de nuevas formas y espacios asociativos, que se desenvuelven en un espacio público que fue, virtualmente, abandonado por lo Estatal.

Hoy los actores sociales reclaman al espacio público, y especialmente al estatal, celeridad, eficiencia y modelos de concertación para resolver demandas sociales con objetivos y métodos legítimos y por tanto consensuados. En ultima instancia lo que está bajo cuestionamiento es el Estado y los actores sociales que sustentaron sus decisiones en base a un proceso de mutua legitimación en donde el respaldo social en las decisiones fue un planteo secundario.

En este sentido, y siguiendo a Castells (1999:394) "es una ironía trágica que cuando la mayoría de los países del mundo han logrado finalmente el acceso a instituciones de la democracia liberal, estas instituciones están tan distante de la estructura y los procesos con importancia real, que aparecen ante la mayoría de la gente como una mueca sarcástica en el nuevo rostro de la historia".

Estos nuevos actores contribuyen a dar sentido a los nuevos valores cívicos, reemplazando formas de solidaridad social y otros valores debilitados por la disputa competitiva que se lleva a cabo entre los individuos que buscaron permanecer en el sistema laboral productivo.

Todo cambio en los valores sociales crea tensiones en la sociedad, que deben ser atendidos por el gobierno local, y van desde la problemática de la familia (desde el extremo uniparental hasta la familia extendida), e incluso los casos de comportamiento anómico propios de la marginalidad, sin dejar de lado la violencia y otros productos de la exclusión y marginación social de grandes sectores de la población.

En la empresa globalizada existe un modelo único de eficiencia, que se impone en todos los mercados, y de este modo la organización social del trabajo impacta y modifica en la organización so- 
cial local, en el contrato social y por ende en el modelo de Estado al que la sociedad tiende. La lógica del mercado globalizado con su necesidad de innovación tecnológica constante y de una acumulación de capitales que lo haga posible son los principales impulsores de este modelo único de eficiencia.

Pero ese mismo proceso puede presentarse como posibilitador, ya que la producción tiende a especializarse, y la eficacia tiende a convertirse en un valor universal. Para los municipios orientar la especialización de la producción a través de la planificación estratégica y una gestión eficiente se vuelve una necesidad que contribuye a una mejor calidad de vida de sus vecinos, siempre que se esté inserto adecuadamente en el sistema productivo mundial, lo que implica redefinir el espacio local, desvincularlo de sus límites territoriales (la clásica circunscripción municipal) e integrarlo en un modelo de relaciones acorde a la naturaleza de los actores globales. La eficiencia a nivel local (en lo gubernamental, lo social y lo económico) puede ser también una forma de atenuar los terribles impactos de la globalización.

No obstante, lo que no se quiere es quedar fuera del circuito globalizador, ser una ciudad o comunidad excluida de los circuitos de producción más rentables. La heterogeneidad que se da en la globalización no afecta únicamente a los individuos sino que puede alcanzar a ciudades, regiones o países enteros.

Las necesidades de los municipios pasan por relegitimar su accionar, por eficientizar su gestión y por generar modelos que le permitan abordar la complejidad y fragmentación de la sociedad glo- balizada. También deberán asegurar la identidad local, para potenciar las acciones colectivas destinadas a insertar a todos los miembros de cada comunidad local en los procesos del mundo globalizado; de este modo puede equilibrarse en algo las tendencias a la fragmentación y exclusión.

Un buen gobierno además de eficiencia administrativa necesita ser transparente, permeable a las demandas de la sociedad que lo eligió, tener una visión gerencial de la sociedad y responder a la sociedad por sus actos. Para ello hay que fortalecer los ámbitos gubernamentales encargados de atender a la población, los ámbitos de participación del sector privado en la formulación de políticas de desarrollo y, por supuesto, a las organizaciones de la sociedad civil que lucha por la integración social (la reducción de las desigualdades).

Para participar del mundo globalizado es preciso que la sociedad civil se haga cargo de algunas funciones que antes estaban depositadas en el Estado, a fin de lograr la eficiencia a través de la participación y el compromiso ciudadano. Resulta menester que la sociedad civil cuente con actores capacitados e independientes que defiendan los derechos sociales y económicos desde una perspectiva de estrategias de largo plazo, que articulen sus acciones en redes sociales que aprovechen las escasas oportunidades que brindan los procesos globalizadores.

Esto exige no sólo un Estado más eficiente, sino un estado inteligente y desburocratizado, que traspase las fronteras que históricamente lo han separado de la sociedad civil y la ciudadanía, capaz de insertar su acción dentro del mercado, y 
que ejerza bien sus funciones privatizadoras y reguladoras, y que promueva activamente la igualdad de oportunidades en estrecha asociación con las organizaciones sociales y con la ciudadanía.

\section{Nuevas realidades}

...La insuficiencia de nuestro antiguo contexto cultural y de nuestras instituciones jurídicas tradicionales para facilitar estos procesos nos obligan a abrir nuevas puertas a la constitución expedita de asociaciones comunitarias o ciudadanas, de redes de protección social o de consorcios entre instituciones públicas y privadas para fines de interés público. Sin embargo permanece la falta de reconocimiento oficial y el respaldo fuerte a un sector público no gubernamental (Bresser et al., 1998).

Surgen sociedades más abiertas, con características diversas, más educadas e informadas, y con grados muchos mayores de movilidad social, por lo que hoy comienza a reivindicarse la importancia de los espacios públicos, de la comunicatividad y del debate, y de que los significados se produzcan en la interacción en el seno de la comunidad, y no sólo en las segmentadas y jerarquizadas esferas en que se desenvuelven hoy la vida privada. Los antiguos ámbitos públicos como la familia, el barrio, la escuela, el maestro, el líder o el club, el aire limpio, las áreas verdes, o la cultura popular, en que se encuentran las afinidades, reemplazadas desde hace tiempo por el consumo que convirtió las anteriores sociedades de ciudadanos en sociedades de consumidores (García, 1995).

En este sentido Quiroga (1996), plantea que estos escenarios nos indican poderosas tendencias asignadas por los cambios en cierne: el quiebre de una cultura estatista y nacionalista, la transformación de un Estado intervencionista y la mudanza de una economía basada en la expansión del sector público por otra apoyada en el libre mercado, la mediatización de la política, el incremento de los umbrales de pobreza, etc. aunque colmadas de imprecisiones y tensiones, los nuevos escenarios prefiguran los perfiles de una nueva realidad.

Toda organización no es más que un conjunto de personas reunidas para satisfacer un interés común. Las sociedades actuales, complejas, pluralistas y abiertas, caracterizadas por su diferenciación y por su multiplicidad de opciones, son sociedades de organizaciones. Un rasgo común a la transformación de los valores que está viviendo nuestro tiempo se refiere al aprecio por la flexibilidad como característica de toda clase de organizaciones económicas y sociales así como también de formas de comportamiento y de vida. Existe un rechazo a las estructuras rígidas de las organizaciones empresariales, políticas y sociales construidas para responder a un paradigma societal en tanto se prefiere como alternativa su estructuración en formas de redes. Tomassini (2000) destaca la opinión de Robert Reich quien subraya esta tendencia como rasgo fundamental del proceso de globalización, y afirma que la fuerza motriz de las actividades económicas y sociales ha dejado de estar radicada en lo que denomina procesos rutinarios o en los servicios personales, para transferirse a las tareas analítico-simbólicas. 
La última parte del siglo $X X$ ha asistido al triunfo universal del mercado que, curiosamente, fue acompañado del triunfo de la democracia. En la situación globalizada, alegan los defensores del nuevo orden, sólo sobrevivirán aquellos países que sean capaces de integrarse a la economía mundial, al tiempo que pongan fin al proteccionismo, al intervencionismo estatal y a la crisis fiscal, pero existe una relación más indirecta, y ciertamente menos percibida, entre la nueva sensibilidad cultural y el tipo de economías adoptadas en la mayor parte del mundo durante el último período. Es entonces entendible que, según Tomassini, el mercado es bueno para la economía, pero no lo es necesariamente para la sociedad, la salud, la educación, la seguridad social, la familia y el desarrollo de las personas.

Lo que torna difícil la gobernabilidad de las sociedades actuales es su evasión respecto a los antiguos modelos que antes la encuadraron, su actual diversidad valórica y su creciente capacidad para elegir entre distintas opciones. La gobernabilidad de la democracia pasa por un reequilibrio entre mercado, la sociedad y el estado, este es el gran desafío del futuro.

Según el autor, las controversias -o las perplejidades- a que actualmente conducen la reflexión o el debate sobre cuestiones éticas se deben a que no sólo han cambiado los valores sino la naturaleza misma de la ética. Esa es la hipótesis de estas reflexiones. La ética es inseparable de la sociedad, está en el corazón de la cultura, que configura la manera de ser, sentir y actuar de las sociedades y de las personas. Nuestros juicios valóricos dependen de nuestra manera de concebir la realidad: la ética depende de la ontología.

\section{El Estado Red}

Como sugiere Castells (1999) los nuevos retos de la globalización sugieren la aparición de un nuevo modelo de Estado: el Estado Red o de redes estatales, que con su soberanía geométrica variable parece ser la respuesta a estos retos del fin de milenio. El Estado Red se caracteriza por compartir la autoridad, es decir, la capacidad de imponer la violencia legitima, con mayor capacidad y extensión que los antiguos estados nacionales.

Así también, al plantearse el modelo en red, supone la existencia de unidades integradas que interactúan favoreciendo los objetivos macro y haciendo frente a desafíos insuperable por unidades menores.

Es evidente que este tipo de Estado-Red, puede verse amenazado por otros Estados-Redes, de igual o mayor tamaño y, en definitiva, el modelo de crisis seguiría funcionando; sin embargo, cuando nos referirnos a los cambios de paradigmas planteamos la necesidad de comprender el paradigma de la sociedad global, en base a modelos de cooperación y solidaridad, en los que el conflicto es el último recurso a tener en cuenta. Esto resulta de entender que en el contexto de los Estados-Redes los actores no son estatales solamente sino, por el contrario, interactúan otros agentes interesados a los cuales el Estado-Red no puede ni debe obviar. 
A la vez se plantea que no todos los actores que integran las redes son iguales; contrariamente, cada uno de ellos tiene características distintivas. El hecho es que estos Estados son interdependientes, de tal modo que ningún actor, ni siquiera el más poderoso, puede pasar por alto al resto, ni aún a los más pequeños, en el proceso integrativo y de toma de decisiones. En el caso de hacerlo, todo el sistema se pone en entredicho y colapsa.

El Estado-Red no pierde soberanía en cuanto a los asunto internacionales, muy por el contrario gana en tanto concede una parte de esta, a cambio de obtener mayor pluralidad de voces en los asuntos que atañen al conjunto que, necesariamente, son los asuntos propios. Temas tales como defensa, economía, ecología, migraciones, empleo, etc son tratados en el conjunto de redes con mayor posibilidad de respuesta a los mismos.

Todo este mecanismo requiere para su funcionamiento la participación, como dijimos, no sólo de los Estados u organismos estatales sino de los nuevos actores antes mencionados por el hecho de que el mismo sea aceptado por los ciudadanos que de ellos participan o se sirven.

En el caso concreto de la Ciudad de Mendoza (Argentina), se han implementado redes a nivel académico con universidades Nacionales y extranjeras logrando el intercambio de conocimientos.

También se han configurado comisiones específicas como la Comisión de Asuntos Transnacionales, sociedad y cultura, en el ámbito del Honorable Concejo Deliberante, en la cual participan desde miembros consulares de diferentes países, asociaciones de inmi- grantes, clubes, empresas, etc, logrando propuestas claras a problemas comunes. Soluciones habitacionales a inmigrantes de países vecinos (caso del Perú, Bolivia, Chile) microemprendimientos cooperativos, convenios de cooperación mutua, etc.

La Ciudad de Mendoza integra lo que se dio en llamar Red de Mercociudades en las cuales participa junto a ciudades como Mar del Plata, Rosario, Montevideo, Córdoba, Santiago de Chile, Río de Janeiro, Sao Paulo, que regularmente asisten a eventos de intercambio y trabajo, alternando las sedes de las mismas en donde las experiencias individuales hacen a la solución del conjunto

\section{La Red de Mercociudades. La nueva cara del mundo}

Hasta el momento hemos planteado que el nuevo orden mundial configura una sociedad con características particulares, signado por nuevos esquemas de organización social, donde la tarea pendiente apunta a normar la convivencia en este nuevo escenario mundial. Asistimos al surgimiento de redes sociales interconectadas que exceden el campo territorial y configuran un nuevo escenario global-regional de acción en el que los asuntos atañen a diversos actores en diferentes lugares del globo; además, desde la cultura global se está valorizando y difundiendo una visión del mundo que privilegia las relaciones entre los temas, las redes existentes entre ellas, por sobre los temas mismos.

Las redes se construyen para reducir las distancias espaciales y tempo- 
rales; la mayoría de las veces nacen del intercambio de información, pero las redes más valiosas son espacios de concertación y de producción, donde cada nodo tiene la misma importancia y las mismas responsabilidades en producir y transmitir.

Las redes transmiten y, si no son conducidas / utilizadas -por cada uno de sus miembros- cuidadosamente, corren el riesgo colapsar: la producción de "basura" puede ser su mayor amenaza. Para esto, la construcción de redes eficientes implica una clara definición de espacios, a fin de facilitar en intercambio de la información; la realización y evaluación de experiencias; la adopción y adaptación coordinada de herramientas de desarrollo social y económico.

Las redes se entrelazan profundamente, y se entrecruzan constantemente; al punto que podemos distinguir rápidamente dos tipos de redes: aquellas que trabajan para la gente y las que trabajan para las redes.

En este contexto, la Red de Mercociudades organizada en base a unidades temáticas que actúan como órganos de la red, que desarrollan un trabajo permanente orientado a objetivos específicos cuyo objetivo más general plantea la integración de las ciudades ubicadas en el espacio regional de los países miembros del MERCOSUR a través de la representatividad y fortalecimiento de sus gobiernos locales. Desde la Red de Mercociudades se ha planteado la necesidad de que la toma de decisiones comience a tener en cuenta la opinión de los propios involucrados.
Este esquema, así como reúne enormes posibilidades, enfrenta grandes desafíos para evitar que se conforme un nuevo espacio socio-económico sin la participación activa de los actores sociales. La Red de Mercociudades entiende que la determinación estratégica de nuclear las realidades de las ciudades del MERCOSUR como herramientas alternativas de adaptación a la evolución de un proceso de integración regional, debe ser apoyada bajo la seguridad de que resulta necesario conocer las mismas para lograr una verdadera integración.

El flujo de información es vital para la construcción de modelos alternativos a los planteados desde la óptica formalista del Estado. La búsqueda de estos nuevos modelos de articulación requiere un estudio pormenorizado de los casos situacionales, en cada área especifica, al igual que las posibles comparaciones fruto de experiencias compartidas en modelos aplicados.

\section{Una reflexión Final}

Es necesario plantearse que, mientras el mundo avanza hacia realidades sin fronteras, la salida a un universo más solidario, mas lógico, en donde prime el modelo de un estado eficiente, con herramientas de gestión capaces de dar respuestas a los problemas sociales, puede plantearse a la red como salida lógica en donde sus hilos unifiquen antes que enredar.

En tal sentido estamos implementando nuevos modelos de gestión, de participación ciudadana, tanto en el Ejecutivo como en el Legislativo local, desde 
todas las áreas interconectadas, junto a los líderes sociales barriales, implementando el estudio de casos, las prácticas de conexión colectiva5, la inserción de la comunidad en los debates de los ámbitos de gobierno, contactos a niveles académicos como jornadas de jóvenes investigadores Argentino-Chilenas, Seminarios de Docentes e investigadores y actores no estatales del proceso de integración regional: en donde la toma de decisiones sea participativa, para que estos espacios de participación ciudadana favorezcan los procesos de integración respetando la identidad cultural de cada actor, generando mecanismos que aseguren que las acciones incidan en la toma de decisiones sin afectar las realidades particulares de cada uno, generando y profundizando nuevos modelos alternativos de gestión que aseguren una producción institucional para atenuar los efectos de un mundo inequitativo y desigual. En definitiva, utilizando las redes para amalgamar soluciones a los problemas de todos.

De este modo se han propiciado diferentes áreas tales como la Unidad Temática de Desarrollo Urbano; Desarrollo Económico Social, Cultura, Género y Municipio, etc. De estas han surgido diferentes proyectos que han generado un intercambio exitoso, entre las diferentes ciudades, en las áreas de bienestar social, cultura, cooperativismo, discapacidad, educación, género, ocupación, salud, entre otras, que están dando nuevas alternativas de abordaje a los problemas que, desde la óptica de la integración, se en- tienden como problemas del conjunto. Como decíamos anteriormente, los problemas de desempleo, marginalidad, contaminación, educación, si bien son observados desde una óptica local, necesitan soluciones globales; en este sentido, el estudio de Mercociudades colabora brindando posiciones comparativas que lleven claridad a los mismos.

\section{Bibliografía Citada}

Bresser Pereira, Luiz C. y otros (1998). "Lo público no estatal en la reforma del Estado". Argentina, Clad, Paidós.

Castells, Manuel (1999). "La Era de la Información. Economía, Sociedad y Cultura”. Vol. 3. España. Alianza Editorial.

García Canclini, Nestor (1995). “Consumidores y Ciudadanos. Conflictos multiculturales de la globalización". México. Grijalbo.

Parker, Crhistian (2000). "América Latina iniciando el 2000: Relaciones Internacionales y Cultura". Seminario Internacional Ciencias Sociales y Humanidades. Santiago de Chile.

Quiroga, Hugo (1996). "Esfera Pública, Política y Ciudadanía. Dilemas de la política democrática Argentina." En Silvia Gaveglio y Edgardo Manero (Comps.): Desarrollos de la teoría política contemporánea. Argentina. Edit. Homo Sapiens.

Tomasini, Luciano (2000). "Cambio cultural, globalización y desarrollo". Chile. Ponencia presentada en el Seminario Internacional de Relaciones Internacionales "Identidad y Cultura", Santiago de Chile. 